\title{
Analysis of Guava Quality by Image Processing
}

\author{
Matheus Pedroza \\ Ferreira \\ Computer Science Department \\ University Federal of Tocantins \\ Palmas/Tocantins - Brazil
}

\author{
Glêndara A. de S. Martins \\ Food Engineering Department \\ University Federal of \\ Tocantins Palmas/Tocantins - \\ Brazil
}

\author{
Warley Gramacho da \\ Silva \\ Computer Science Department \\ University Federal of Tocantins \\ Palmas/Tocantins - Brazil
}

\begin{abstract}
Brazil is an important fruit producer in the world. Despite the enormous production, fruit classification techniques do not follow the requirements of consumer protection institutions regarding food quality, since visual and manual classification techniques are still widely used. In this way, the development of machinery and grading systems has been increasingly exploited in order to meet the market's demands. The objective of the present study is to develop a system capable of identifying defects on the guava surface to determine its degree of quality.
\end{abstract}

\section{General Terms}

Computational vision, image processing and segmentation

\section{Keywords}

Guava, Quality, Classification, System applied.

\section{INTRODUCTION}

Brazil is the world's third largest fruit producer, followed only by China and India [1] being responsible for 5\% of world's production in 2010 (IBRAF), which generates an annual income of $\mathrm{R} \$ 10$ billion.

Besides the enormous production, the fruit classification techniques do not follow the requirements of consumer protection institutions regarding the food quality, since visual and manual classification is still broadly utilized [2]. The authors say that fruit selection and classification is something that needs national scope development, because a human decision is subjective and may be faulty, culminating in the quest for postharvest automation. Thus, the development of machines and classification systems is increasingly explored to meet the marketplace requirements regarding food quality.

One of the notable classification techniques that have been outstanding for the effectiveness shown in non-destructible extraction and quantification of characteristics related to the quality and control of fruits is the computational vision. Its main goal is to promote a more objective way. According to [3], the computational vision is the science concerned with the study of the theoretical and algorithmic basis by which the useful information regarding a respective object or scene can be automatically extracted and analyzed from an image.

For a long time, the agroindustry is trying to automatize the process of fruit selection to increase productivity and quality of production. The computational vision provides an automatized solution for what was done visually. Techniques currently present on market can calculate parameters as size [4], color, shape or degree of maturation [5] and defects or stains $[6,7]$.

In digital image processing, the segmentation procedure of the image influences the success of the algorithm utilized for the analysis. Segmentation is defined as the process subdivision of images in regions and objects of interest [8]. There are several methods currently utilized in industry for image segmentation, each one with its own attractive characteristics for a determined type of problem.

The choice of the best algorithm for segmentation of a given scene depends on the restrictions imposed by the problem being solved. In the present case, the automatic inspection of fruits is done in a very short time gap [9], which restricts the precision of the algorithm to reach an acceptable performance.

Some of the most famous techniques are based only on the similarity of the pixels expressed in three-dimensional coordinates to classify the image. Therefore, it is necessary that the system knows how to differentiate pixels in distinct regions of the image. In this way, a specialist is responsible for the process named 'training', which is nothing more than relating in order to be implemented in the system the different possible regions of the image and the value of the pixels in these regions.

There are different methods oriented to regions, which take as criteria for classification different regions of the image and its properties. This type of method uses the information present in the neighborhood of a pixel for its classification. Because it uses the information present in the image as basis for segmentation, this type of algorithm does not require a previous 'training', making unnecessary the presence of a specialist in the process.

Another factor that makes this type of method very attractive to industry is the high adaptation the external changes. Even if the change of the fruits of some harvest may be abrupt, the performance of the algorithm and the quality of the results should not be affected. Some of these algorithms can even be used on different types of fruits, changing only some specific constants.

The present work is based on an application developed in [10] that classifies citric fruits using several techniques of computational vision.

\section{MATERIAL AND METHODS}

The system based on computational vision was developed following several steps, divided into three groups as described in [3]: image acquisition, image processing and image analysis.

\subsection{Image acquisition}

The analyzed fruits were obtained in marketed or supermarkets in Palmas - Tocantins. At first, the only restriction imposed on the fruit images to be classified is that the background of the image must be uniform and distinguishable from the fruit and any other present defect. 


\subsection{Image Processing}

The system is built from some algorithms tested on several types of images. For a better precision, each algorithm was developed individually, comparing its outputs with the results presents in the literature.

It is important to stress that the algorithms developed along the project are not only applied to the classification of fruits, but to the segmentation of general images. At the end of the project, some modifications were mad to direct the algorithm to the analysis of guavas, to increase its performance.

The process consists in the use of an initial algorithm to filter the image, removing noise and smoothing color differences between neighboring pixels [11]. Following the filtering process, a quantization algorithm is applied, drastically reducing the number of colors in the image [12]. The final step of segmentation adopts as criteria for the classification the partitioning of the image according to the results of the quantization [13].

\subsubsection{Smoothing and noise removal}

The first step following the acquisition of the images that will be computationally analyzed consists in the elimination of noises generated from the image gathering. In some cases, as in the process described here, the elimination of divergences of the color of the pixels of the same region is also necessary. It is necessary that conglomerates of pixels exhibit uniform colors so they can be classified into distinct regions. In the case of pixels of the same region show significant differences in their colors, they will not be classified as belonging to the same region.

Usually, in this task, the Gaussian filter, median filter or the Vector Median Filter (VMF) [14] are used. The filtering by these methods generates uniform areas along the entire image reducing, however, the amount of details in the image. Small defects in the fruits might be wiped out in the application of these algorithms.

To contour this problem, the chosen method was the Peer Group Filtering (PGF), described in [10]. As in the above methods, the PGF consists in convolving the entire image with a kernel and then calculate the new value of the pixel according to the convolution in each point. However, unlike the other methods, the PGF only considers similar pixels along the kernel, smoothing only pixels of the same region and preserving most of the details of the image.

\subsubsection{Quantization}

The quantization step consists in the reduction of the number of colors in the image. In the RGB space, the total number of representable colors is $2^{\wedge} 24$. The segmentation used in this work has its performance direct related with the number of colors in the image. If all the colors would be considered, the processing time of the segmentation would be infeasible.

The final number of colors at the end of the quantization was established to 32. In [10] it was observed that the results are very similar with 32,64 and 128 colors. As the running time is decreased with a lesser number of colors, the value was set to 32 .

The chosen algorithm was the k-means, which is between the most used algorithms for clustering [12]. The k-means is a heuristic developed by Stuart P. Lloyd [16] that requires a set with $\mathrm{K}$ initial centroids, usually chosen randomly [17]. At each iteration, the value of the center of each set is applied to represents all the points belonging to it. The center of each set is then recalculated as the mean of all the points of the same set. These steps are then repeated until convergence [12].

The chosen method of initialization was the Variance-based method (WAN) [18], presented as one of the methods with the best results.

\subsubsection{Segmentation}

The method used for segmentation is described in [13] as JSEG. The algorithm consists initially in the separation of the image in classes, by means of some quantization technique. Each color resulting from the quantization process is utilized as a class to build a class map that is then used in the segmentation process.

The JSEG consists in the minimization of a cost associated with the partitioning of the image in classes. The value of each point in the class map is given by its position in the image as a bi-dimensional vector $(x, y)$. Let $Z$ be the set of all the $N$ points in a class map and $Z_{i}$ the set of all the points belonging to class $i$. Let $z=(x, y) z \in Z$ and $z_{i} \in Z_{i}, m$ the mean of all the points of the image and $m_{i}$ the mean of class $i$ :

$$
m=\frac{1}{N} \sum_{z \in Z} Z \quad m_{i}=\frac{1}{N_{i}} \sum_{z \in Z_{i}} Z
$$

Let $S_{t}$ be the total variance in the entire image defined as:

$$
S_{t}=\sum_{z \in Z}\|z-m\|_{2}^{2}
$$

And $S_{w}$ the total variance regarding the points belonging to the same class:

$$
S_{w}=\sum_{i=1}^{C} S_{i}=\sum_{i=1}^{C} \sum_{z \in Z_{i}}\left\|z-m_{i}\right\|_{2}^{2}
$$

Therefore, the value $J$ to be minimized is given by:

$$
J=\left(S_{t}-S_{w}\right) / S_{w}
$$

It is possible to realize that $J$ is always positive because it is always true that $S_{t} \geq S_{w}$. If the image is composed solely by several homogeneous regions, the classes will be more dispersed and the value of $J$ is high. If all the classes are uniformly distributed along the entire image, $J$ is small. The idea behind $J$ value came from Fisher's Linear Discriminant [15].

The value of $J$ is a good indicator of the homogeneity of a region. The next step consists in the use of circular windows of several scales to determine possible regions in the image. $J$ is then recalculated for each region defined for the window and the median of all the values of $J$ along the image is given by $\breve{J}$ :

$$
\breve{J}=\frac{1}{N} \sum_{i} M_{i} J_{i}
$$

Where 
$J$ calculated along the region $i, N$ is the total number of points in the image and $M_{i}$ the number of points in

the region $i$.

The criterion for segmentation is then minimize $\breve{J}$ over all regions. Therefore, it is necessary the creation of "seeds" over all image, representing possible homogeneous regions. A threshold value $T$ is used to create the seeds, and is given by:

$$
T=\mu+\alpha \sigma
$$

Where $\mu$ is the mean of the values that represents the homogeneity (values) over all image and $\sigma$ is the standard deviation. $\alpha$ is a constant whose value is chosen by tests and depends entirely on the type of the image.

Pixels with $J$ values greater than $T$ are considered possible seeds and are grouped together according with 4-connectivity $(\{(\mathrm{x}+1, \mathrm{y}), \quad(\mathrm{x}-1, \mathrm{y}),(\mathrm{x}, \mathrm{y}+1),(\mathrm{x}, \mathrm{y}-1)\}$, where $(\mathrm{x}, \mathrm{y})$ is the position of the pixel analyzed). Areas with more than 32 pixels (approximately $1 \mathrm{~mm}^{2}$ ) are set to be seeds [10].

After the definition of the initial seeds, the process of region growth starts. At each iteration, the pixels that were not chosen to be seeds are set to be part of some neighboring seed according to a criterion based on the value of $J$. The process ends when all the pixels belong to some seed.

At the end of all the process, the image will present more segmented regions than the optimal value. To contour this problem, an agglomerative algorithm [20] is used. The adopted criterion consists in the calculation of the distance between histograms of different adjacent colors in the image:

$$
D(i, j)=\left\|P_{i}-P_{j}\right\|_{2}
$$

Where $P_{i}$ is the histogram vector of colors in the region $i$. At each iteration, the regions that present the smaller value of $D$ are joined to form a single region. The process continues until

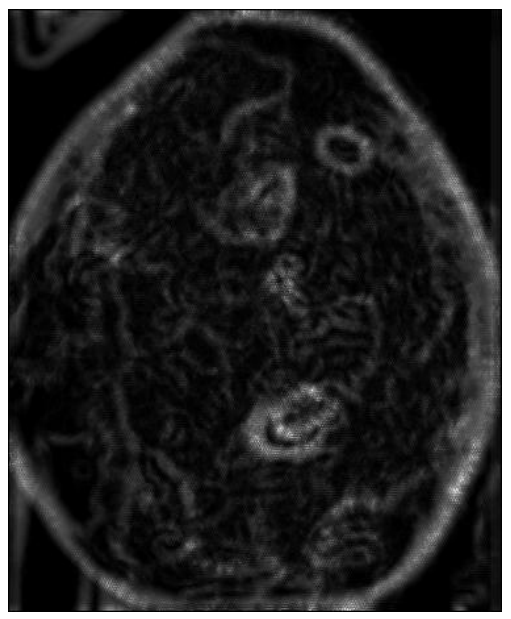

(a) the smaller of the distances be greater than a defined threshold $T m$, determined by the user.

\section{RESULTS AND DISCUSSION}

The algorithm for quantization uses the colors generated by the quantization as classes to define $J$ values for each pixel in the image. Each pixel has a value according to its local homogeneity, defined by the classes of the neighboring pixels. Pixels in boundary regions have greater values, while pixels in homogeneous regions have their value close to 0 . The image then becomes a three-dimensional terrain: each pixel is represented by its position $(\mathrm{x}, \mathrm{y})$ and its $J$ value, which represents peaks (boundary regions) and valleys (homogeneous regions).

The size of the window influences directly in the $J$ values of each region. Windows of larger size (64x64 as described in [13]) determine larger homogeneous regions in the image, while smaller windows determine small objects or boundary regions (Figure 3a).

Overall tests, the application of a single window of dimension $7 \times 7$ proved to be superior to the use of windows of larger size or even more than one window (Figure $3 b$ ). This is due to the fact that most of the defects presents on the surface of guavas have a size too small to be found by windows of larger size, but if the window size is too small, boundary regions of larger size would be lost. In [10] a single square window of dimension $3 \times 3$ was used, because the analysis of some defects of small size (approximately 32 pixels $-1 \mathrm{~mm}^{2}$ ) are impossible with windows of larger size.

Bellow follows a graphical representation of the $J$ values generated by the application of windows of different sizes. The values were normalized between $0-255$, so they can be represented on gray level. Darker areas have smaller $J$ values and are defined as homogeneous regions, while brighter areas have greater $J$ values and represent boundary regions.

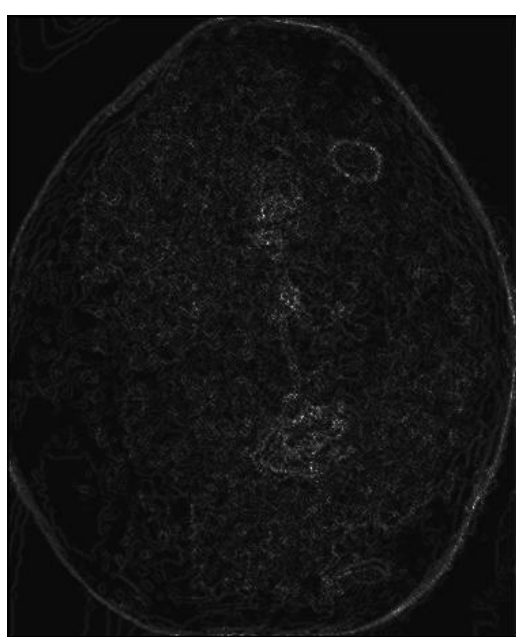

(b)

Figure (3): (a) result from the application of a $64 \times 64$ window, (b) result from the application of a $7 \times 7$ window. $J$ images normalized for graphical representation.

It is possible to observe by the images that windows of larger size delimit areas of larger size better than small windows, but they fail in delimiting precisely small regions (Figure 3 a and b). Therefore the use of windows of large size in the segmentation of defects on the analyzed fruits is unfeasible. 
Even with reduced sized, the $7 \times 7$ windows can delimit large areas (as the contour of the guava) and at the same time can delimit precisely small areas too.

The next algorithm determines possible seeds according to a threshold that depends on the $J$ value over the entire image. These seeds are then expanded being connected (4connectivity) to neighboring pixels that are bellow that same threshold. Regions generated by this expansion that has size greater than the established (32 pixels in the case) are determined seeds. At the end of the process, each seed formed represents a segmented object in the image.

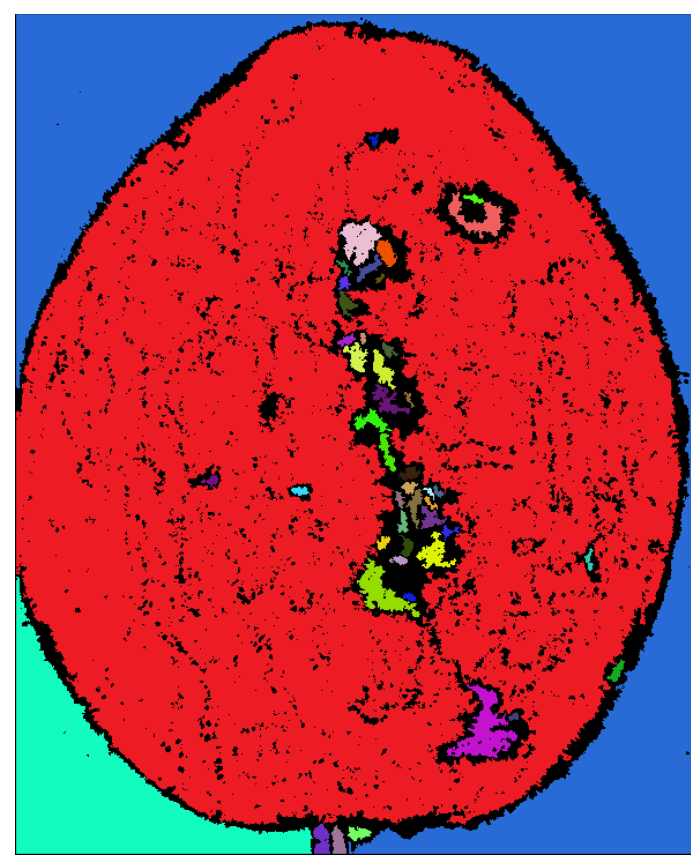

(a)
The number of segmented objects, in most of the time, is greater than the expected number. This is due to the segmentation of uniform areas in the image in more than one region. A conglomerative algorithm [16] is then used to join the regions according to its color, determined by the original image.

To represent this result graphically, a random color was set to each object, so the boundary of each region is clearly shown. An example follows with a window of dimension 7x7 (Figure 4).

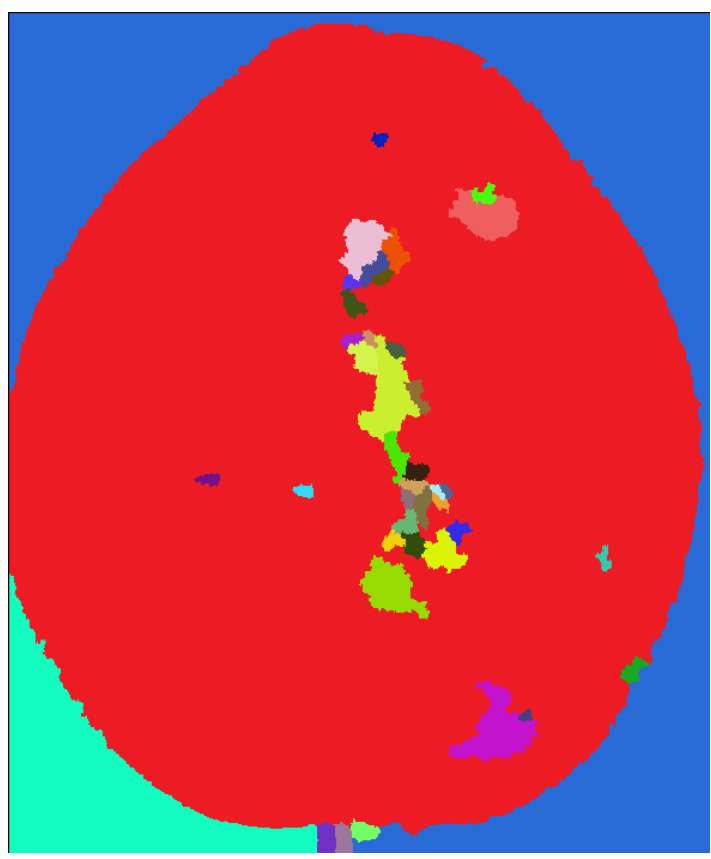

(b)

Figure (4): (a) the result from the creation and expansion of seeds, (b) the result from the process of merging. Images shown with random colors, with one distinct color to each segmented region. The background of the image was considered purposely to the illustration of its total segmentation. Two distinct regions, as expected, were formed at the background due to the 4connectivity.

Following the application of the conglomerative algorithm, the segmented regions were delimited for the visualization of the results. The delimited areas are distinct regions, each one representing a different object in the image. At the end of the process, the region that shows the largest uniform region is necessarily the surface of the fruit, and the small regions are the fruit defects.

It is important to address that the background of the image was kept purposely during all the execution of the algorithm. In a real system, it is utmost important that the image background is totally identified so the results are not affected. In the presented examples, the background of the image in all the cases is completely identified (even with sharp differences of luminosity and color, even within the same image) and generates regions distinct from the fruit and its defects.
It is clear that the system can segment several types of defects, with different colors and sizes, independent from the color of the image background or the luminosity of the environment. Changes in the tonality of the surface of the fruit impact almost nothing in the segmentation of defects. As proposed, the system is extremely robust regarding changes in the environment and can provide good quality results without the specification of parameters such color, luminosity or changes in the tonality, for example.

Figure 5 shows one more example comparing the original image with the final segmentation. 


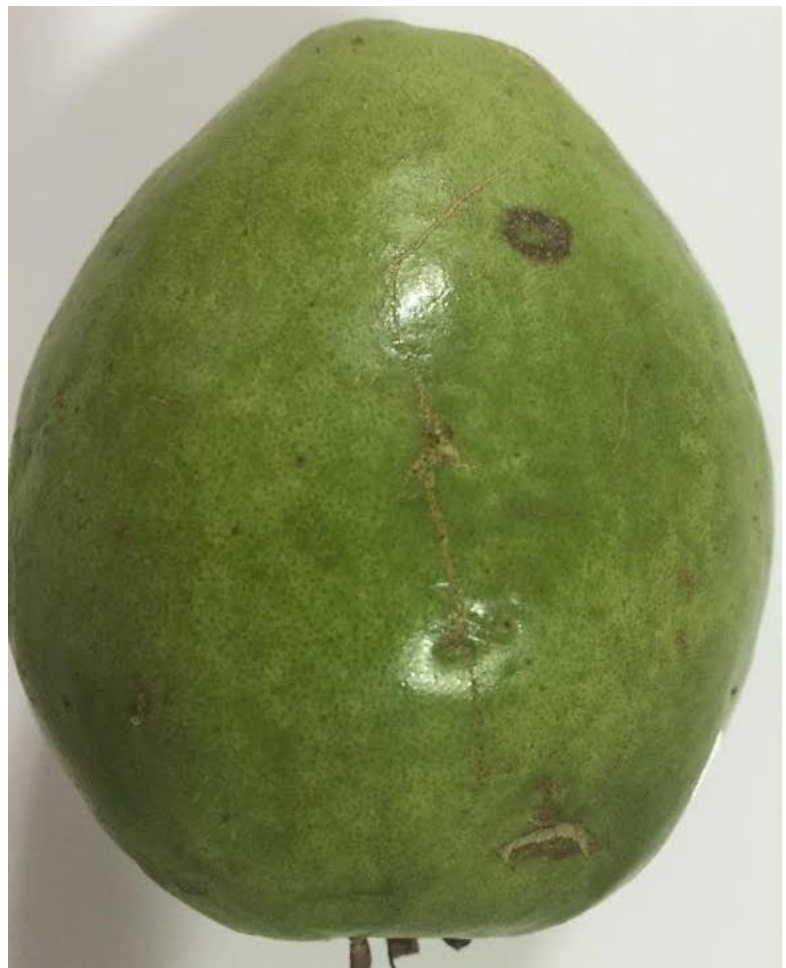

(a)

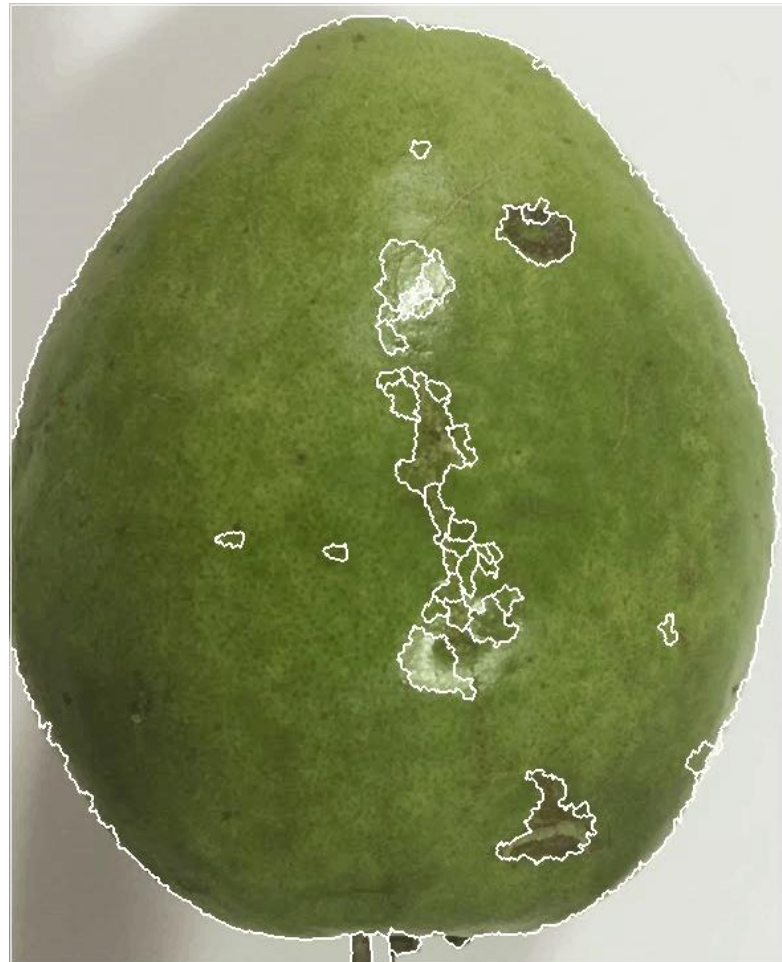

(b)

Figure (5): (a) original image, (b) final result of segmentation. Segmented regions defined by white contour.

The above image has defects of different color, dimension, and texture. Despite the differences between the defects found on the image, the system is capable of detecting each one of them, with a very small number of false positives (segmented regions that actually does not represent distinct regions).
The following examples (Figure 6) show that fruits with completely different colors and even several fruits on the same image can be segmented with good quality by the system with no tuning between each run.

\section{Original Image}

Smoothing and Noise Removal

Quantization
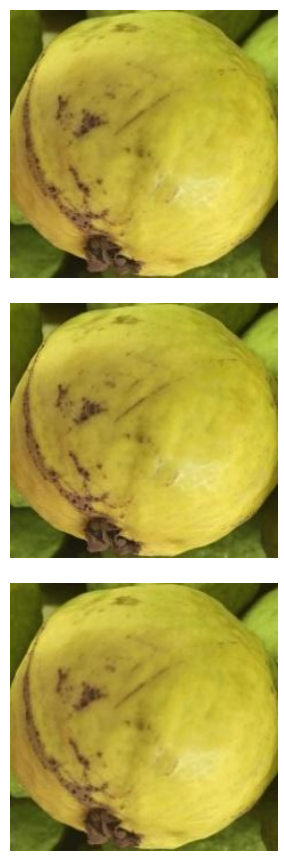
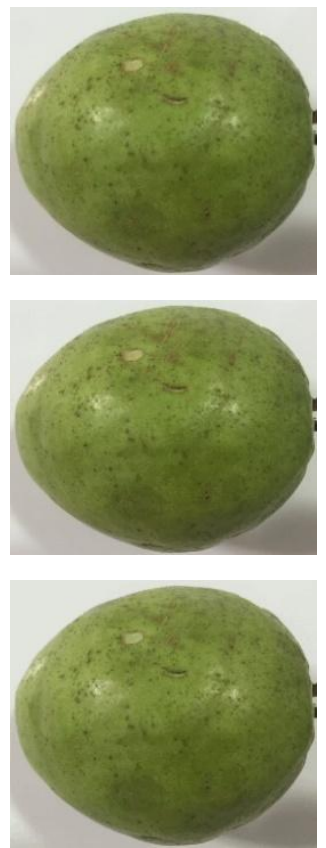
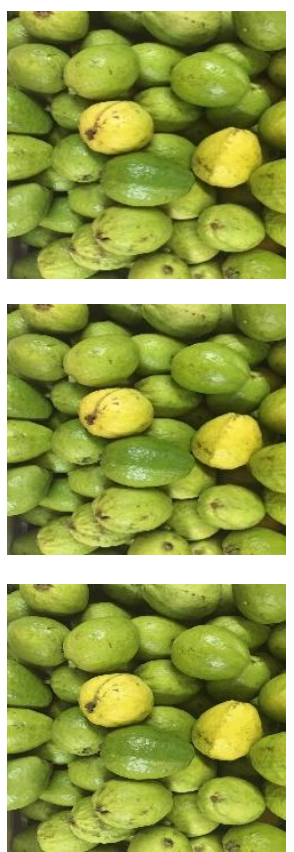
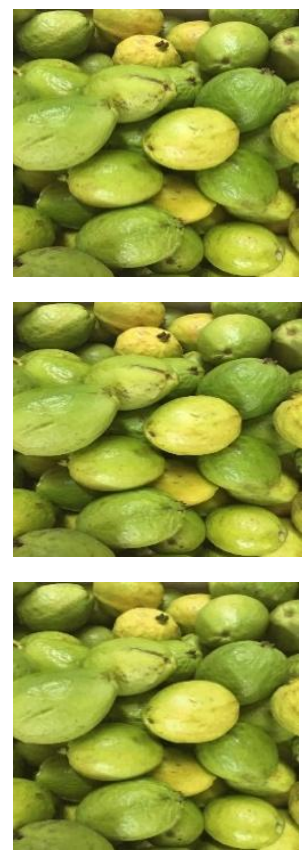
Jvalues
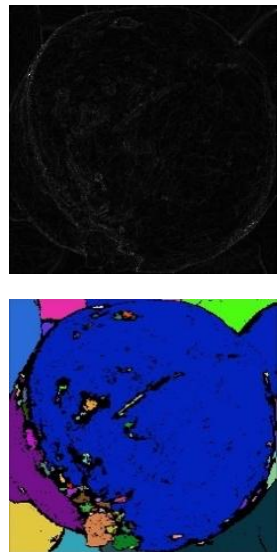

Seeds

Creation

(random

colors)

Complete

Seeds

(random

colors)

Merging

(random

colors)

Segmentation
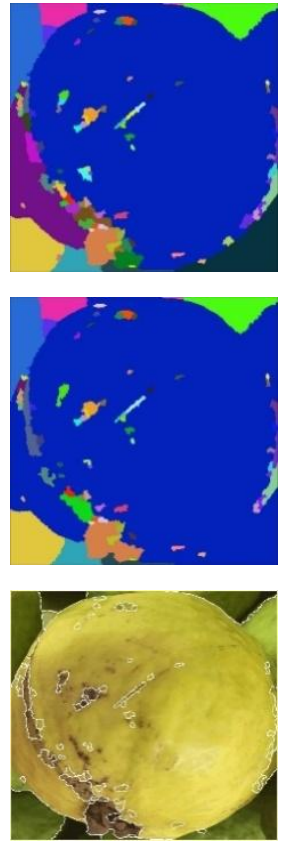
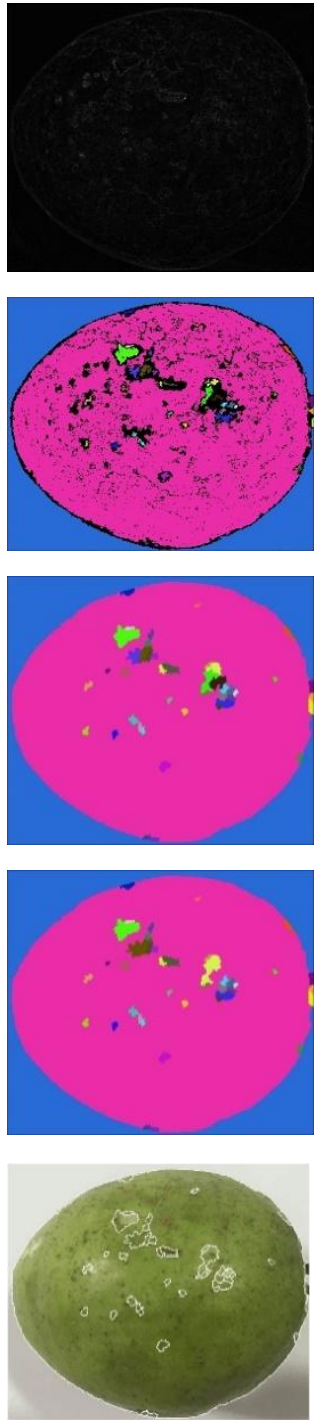
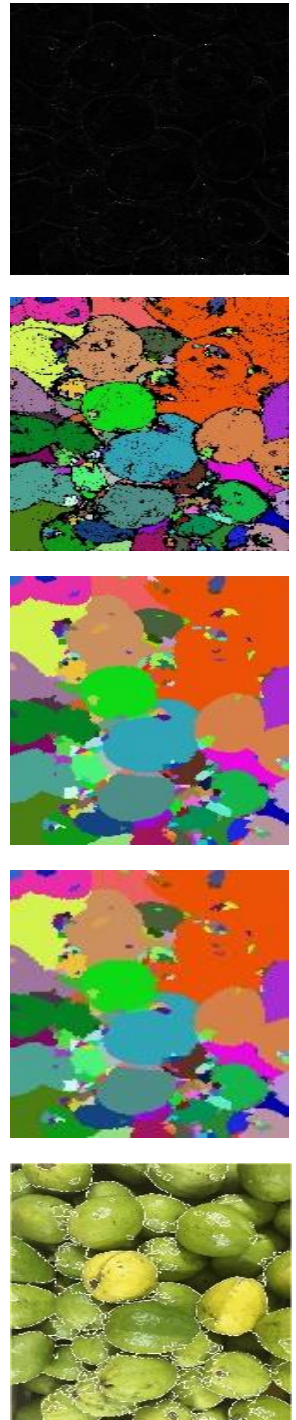
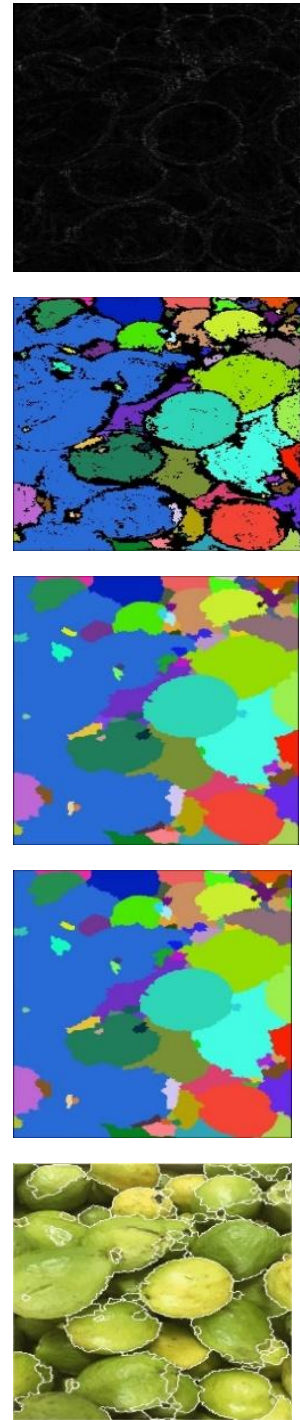

Figure 6: Example for fruits with completely different colors

\section{CONCLUSIONS}

The segmentation model presented here fits very well with the goal of the project. The running time of the algorithm is completely feasible to real time classification (order of tenths of seconds on an average computer), just as it was initially proposed.

The system is very robust regarding the changes on the analyzed images. Different fruits, with different characteristics (color, degree of maturation, size) were tested with the exact same parameters, showing an efficient segmentation of the defects.

It is important to stress that the choice of guava has impact only in the definition of the parameters of the algorithm, as the constant $\alpha$ (seed determination) and the threshold $\mathrm{Tm}$ (seeds merging, as in the choice of the number and dimension of the windows. It is possible to obtain a very good segmentation choosing specific parameters for the type of image to be analyzed without a single modification to the algorithm. In this way, with defined parameters, the system is capable of analyze fruits with completely different characteristics (or even general images).

Despite the robustness regarding the analysis of general images, the algorithm is very sensitive to changes in the parameters. To obtain a good quality segmentation, it is essential to define specific parameters for each type of image to be analyzed.

The main problem with the algorithm resides on the incapacity of automation on the process of parameter selection. In [10], the authors discuss the hardness in establishing a method of parameter selection. In [13] the parameter was chosen by experimentation, as in this work.

Anyway, the optimal value of the parameters is defined entirely by the image characteristics. A future work might analyze some method capable of optimize the value of the parameters according to the characteristics of the analyzed images. Such method could provide a generalization regarding the use of the algorithm on distinct images and lead to a possibly significant increase in the final performance of the segmentation, given the dependency of the algorithm on the value of the parameters.

\section{ACKNOWLEDGMENTS}

Our thanks to CNPq for financial support in the execution of this work. 


\section{REFERENCES}

[1] Agência Sebrae. 2009. Brasil é o terceiro maior produtor de frutas do mundo. Disponível em http://www.canalrural.com.br/canalrural/jsp/default.jsp?u $\mathrm{f}=1 \&$ local $=1 \&$ action=noticias\&id=2535814\&section=no ticias. Acesso em 06/03/2014

[2] Rodrigues, J.C.; Filho, J.M.L. Análise de qualidade de frutas por imagens multiespectrais. Revista Científica Eletrônica UNISEB, v.1, n.1, 91 - 110p., 2013.

[3] Panigrahi, S.; Gunasekaran, S. Computer vision. In: GUNASEKARAN, S. (Ed.). Nondesctructive food evaluation: techniques to analyze properties and quality. New York: Marcel Dekker, 2001. p. 39-98.

[4] Brodie, J. R., Hansen, A. C., and Reid, J. F. (1994). Size assessment of stacked $\operatorname{logs}$ via the Hough Transform. Transactions of the ASAE, 37(1), 303-310.

[5] Diaz, R., Faus, G., Blasco, M., Blasco, J., and Molto, E. (2000). The application of a fast algorithm for the classification of olives by machine vision. Food Research International, 33, 305-309.

[6] Aleixos, N., Blasco, J., Navarron, F., and Molto, E. (2002). Multispectral inspection of citrus in real-time using machine vision and digital signal processors. Computers and Electronics in Agriculture, 33(2), 121137.

[7] Leemans, V., Destain, M. F. (2004). A real-time grading method of apples based on features extracted from defects. Journal of Food Engineering, 61(1), 83-89.

[8] Gonzalez, R. C., Woods, R. E. (2002). Digital image processing. NJ, USA: Prentice Hall.

[9] Aleixos, N., Blasco, J., and Molto', E. (1999). Design of a vision system for real-time inspection of oranges. In
VIII national symposium on pattern recognition and image analysis (pp. 387-394). Bilbao, Spain.

[10] J. Blasco, N. Aleixos, E. Molto (2006). Computer vision detection of peel defects in citrus by means of a region oriented segmentation algorithm.

[11] Yining Deng, Charles Kenney, Michael S. Moore and B. S. Manjunath (1999). PEER GROUP FILTERING AND PERCEPTUAL COLOR IMAGE QUANTIZATION. Department of Electrical and Computer Engineering, University of California.

[12] M. Emre Celebi (2011) .Improving the Performance of K-Means for Color Quantization. Department of Computer Science, Louisiana State University.

[13] Yining Deng and B. S. Manjunath (2001). Unsupervised Segmentation of Color-Texture Regions in Images and Video.

[14] J. Astola, P. Haavisto, and Y. Neuvo, "Vector median filters”, Proc. of IEEE, vol. 78, p. 678-89, April, 1990.

[15] C. Kenney, Y. Deng, B. S. Manjunath, and G. Hewer (2001) .Peer Group Image Enhancement.

[16] R.O. Duda and P.E. Hart, Pattern classification and scene analysis, John Wiley \& Sons, New York, 1970.

[17] S. Lloyd, Least Squares Quantization in PCM, IEEE Trans. on Information Theory 28 (2) (1982) 129-136.

[18] S. Wan, P. Prusinkiewicz, S. Wong, Variance-Based Color Image Quantization for Frame Buffer Display, Color Research and Application 15 (1) (1990) 52-58.

[19] Cofilab, (2014). Image database 'Apple Golden'. Retrieved from http://www.cofilab.com/portfolio/goldendb/ 